\title{
SPECTRAL REFLECTANCE MEASUREMENT METHODOLOGIES FOR TUZ GOLU FIELD CAMPAIGN
}

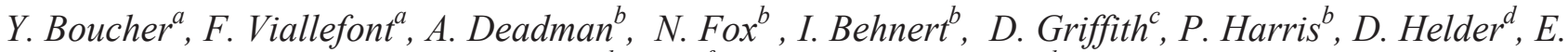

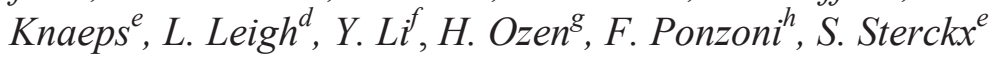 \\ ${ }^{a}$ Onera - The French Aerospace Lab, F-31055 Toulouse, France \\ ${ }^{\mathrm{b}}$ NPL - National Physical Laboratory, Teddington TW11 0LW, United Kingdom \\ ${ }^{c}$ CSIR - South Africa Council for Scientific and Industrial Research, Pretoria 0001, South Africa \\ ${ }^{\mathrm{d}}$ SDSU - South Dakota State University, Brooking SD 57007, USA \\ ${ }^{\mathrm{e}}$ VITO - Flemish Institute for Technological Research, $2400 \mathrm{Mol}$, Belgium \\ ${ }^{\mathrm{f}}$ National Satellite Meteorological Center China Meteorological administration, Beijin 100081, China \\ g TÜBITTAK UZAY - Space Technologies Research Institute, 06531 Ankara, Turkey \\ ${ }^{\mathrm{h}}$ INPE - National Institute for Space Research, 12227-010 São José dos Campos-SP, Brazil
}

\begin{abstract}
A field campaign had been organized in August 2010 on Tüz Gölü salt lake, Turkey, with the aim of characterizing the site for satellite optical sensor vicarious calibration, and of comparing different methodologies of surface reflectance factor characterization. Several teams have made groundbased reflectance measurements with a field spectrometer on different areas of the salt lake of $100 \mathrm{~m} \times 300 \mathrm{~m}$ and $1 \mathrm{~km} \mathrm{x}$ $1 \mathrm{~km}$ size. Different types of sampling strategies and measurements methods have been used by the participants, and are described in this paper. Preliminary results on one area are presented, that show a good agreement between the different measurements.
\end{abstract}

Index Terms - Vicarious calibration, reflectance, salt lake

\section{INTRODUCTION}

The aim of vicarious calibration [1] is to verify the postlaunch radiometric calibration performance of satellite optical sensors, by the comparison of the radiance measured on-orbit and the one propagated from the optical or radiative properties of the measured reference test site. The objective of the 2010 CEOS Key comparison [2] was to identify and evaluate any biases in instrumentation used by participants and variances in the results of using different methodologies for reference site characterization, in this case Tuz Gölü test site. Two different concepts can be used for vicarious calibration of satellite optical sensors. The radiance-based methods use upwelling ground-based radiance measurements, simultaneous to the satellite acquisition, propagated to the sensor entrance pupil with a radiative transfer code. The simultaneity is essential, so that a small area can be measured only in a short time, and the uncertainty associated with the radiance measurement, which can be rather poor, is directly propagated to the calibration performance. The reflectance-based methods use groundbased reflectance measurements, which have to be done with illumination conditions as close as possible to the ones of the satellite acquisition, and the same viewing angle. These latter reflectance methods provide more detailed and accurate site characterization, which should improve the accuracy of the radiance propagated to the entrance of the sensor. Thus, the objective of this paper is to describe and compare the ground-based reflectance measurements methods used by the different teams during Tuz Gölü field campaign.

Section 2 presents the principle of field spectral reflectance measurements. It describes the practical details and considerations contained within the choice of sampling strategy and measurement redundancy, and ends on practical instrumental considerations. Section 3 presents the methodologies of reflectance measurement of all the teams, which can be grouped in classes: 1) spaced out sampling with redundancy, 2) space out sampling with variability evaluation, and 3) in-motion sampling. Section 4 presents details of an exercise to cross-compare the results produced by different teams when sampling the same $50 \mathrm{~m} \times 3 \mathrm{~m}$ site but using their specific sampling methodology. Section 4 also includes preliminary results from one site together with some discussion of the results.

\section{CONSIDERATIONS ON REFLECTANCE FIELD MEASUREMENTS}

\subsection{Principle of field spectral reflectance measurement}

Since the aim of these measurements is the vicarious calibration, radiance and reflectance ground measurements 
must be spectrally resolved, to allow a precise integration to match the satellite sensor bands. Moreover, more detailed spectral measurements allow their use for a wider range of sensors.

In the field, the signal $\mathrm{S}_{\text {ground }}$ given by the radiometer looking at the ground surface is measured first at time $t_{1}$, then the signal $\mathrm{S}_{\text {ref }}$ given by the radiometer looking at a reference panel (whose reflectance $R_{\text {ref }}$ is known) is measured at time $t_{2}$. The reflectance of the ground is [3]:

$$
\mathrm{R}_{\text {ground }}=\mathrm{R}_{\text {ref }} \times \mathrm{S}_{\text {ground }}\left(\mathrm{t}_{1}\right) / \mathrm{S}_{\mathrm{ref}}\left(\mathrm{t}_{2}\right)
$$

Implicitly, the hypothesis is that the solar irradiance did not change between $t_{1}$ and $t_{2}$, and this requires that both measurements are carried out within a short time interval. The global irradiance is a combination of direct and diffuse solar light. The incident angle of the direct light is defined by the sun position in the sky, and the viewing angle is chosen by the operator (the measurement is often done at nadir viewing). Thus, the measured reflectance is a combination of hemispherical-directional and bi-directional types. The reflectance $\mathrm{R}_{\text {ref }}$ of the reference panel can be bidirectional, or directional-hemispherical. Most of the reference panels used in the field are Spectralon or Spectralon-like panels, thus their behavior is close to the behavior of a Lambertian surface. Except for very large solar or viewing angles, it is not mandatory to introduce a BRDF correction for the reflectance factor of the reference panel. However, it's possible to take this into account in the uncertainty budget.

\subsection{Instrumentation and sampling area}

All of the teams except INPE used a Fieldspec ASD spectroradiometer. In this case, the user has to choose the aperture of the objective and the ASD configuration (the number of elementary spectra averaged to get one measurement, here typically 10 , and the number of dark current acquisitions, here typically 25). The spectroradiometer must also be optimized from time to time, to avoid saturation and/or bias on the measured signal.

If the site characterization is intended to be used for spaceborn sensor calibration, the ground area sampled by the spectrometer could be as large as the sensor pixel. The area sampled increases with the objective aperture and focal length (leading to field of view) and the height above the surface being measured. For practical reasons, the height is limited to that of a human being. A field of view of $8^{\circ}$ has been used by most of the teams, viewing the site at nadir and sampling a surface of about $15 \mathrm{~cm}$ in diameter. Nevertheless, for some measurement scenarios, the size of the reference panel limits the height or the field of view and thus the size of the ground area under analysis.

INPE used a CIMEL 313 field multispectral radiometer: 5 bands in the blue, green, red, near IR (800-900nm) and SWIR (1550-1700), with a $10^{\circ}$ field of view.

\section{PRESENTATION OF REFLECTANCE MEASUREMENT METHODOLOGIES}

The pixel size of most satellite sensors is relatively large, but to ensure a good accuracy several pixels have to be sampled and considered for the calibration. The areas to be characterized such as $300 \mathrm{~m}$ x $100 \mathrm{~m}$, are very large compared to the spot size sampled by the ASD instrument. This requires a sampling strategy to be defined aiming to give a good assessment of the mean reflectance of the area and an idea of the variations around this mean. As explained in the previous section, the global time of measurement of one area has to be short enough to have no significant change in the illumination conditions. In this case the target was to not exceed one hour. The measurement methodologies used can be divided into three classes according to three sampling strategies.

\subsection{Spaced out sampling averaging several measurements over one point}

This sampling strategy is done according to a regular grid with spaced out sampling points. At each point of the grid, several measurements are averaged which allows evaluation of the type A uncertainty [4]. Thus, the variation of the reflectance between the different points is a combination of the variation at small scale and at the scale of the sampling grid, typically between $20 \mathrm{~m}$ and $40 \mathrm{~m}$. This strategy has been chosen by Onera (Fig. 1), Tübitak Uzay (Fig. 2), CSIR (Fig. 3), CMA (Fig. 4) and INPE (Fig. 5). Onera averaged 10 measurements per point, with a measurement on the reference panel (5 averaged) before and after. TU averaged 10 measurements per point, with an optimization and reference measurement every 10-15 minutes. CMA averaged 5 measurements per point, reference measurements being performed before and after.

\subsection{Space out sampling with estimation of the local spatial variability}

This sampling strategy is similar to the previous one, excepting that for each measurement point, several measurements are locally recorded over different points near the nominal location.

This sampling approach allows an evaluation of both local and overall spatial variability of the site reflectance. It was followed by VITO and is represented in Fig. 6 for $300 \mathrm{~m} \mathrm{x}$ $100 \mathrm{~m} \mathrm{(a)} \mathrm{and} 1 \mathrm{~km} \mathrm{x} 1 \mathrm{~km}$ (b) sampling sites. At each location, spectral reflectance measurements were performed over four distinct sampling points (Fig. 6c), and four measurements averaged for each point. Spectralon reference measurements were performed before and after these repetitions. However, it turned out to be a relatively time consuming method, strongly limiting the number of transects to be finished within the foreseen timeframe of one hour. 


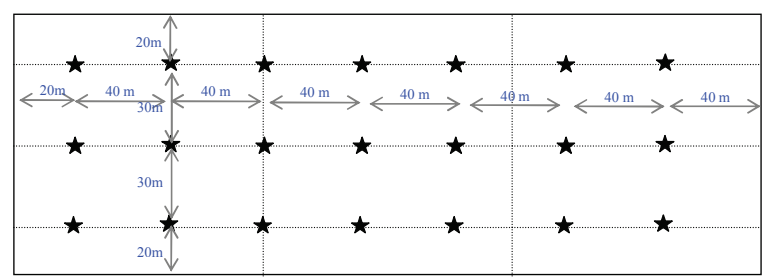

Fig. 1: Onera's sampling points for $300 \mathrm{~m} \times 100 \mathrm{~m}$ areas

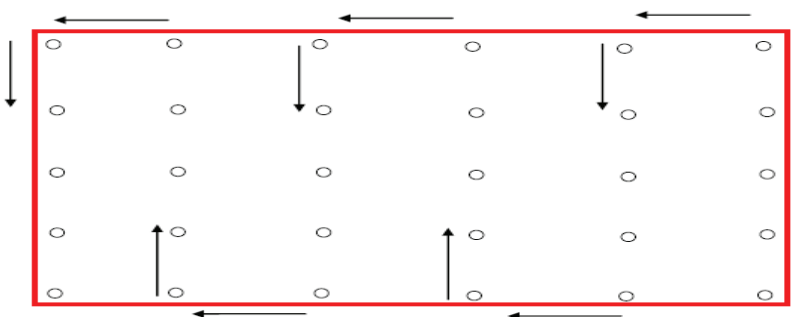

Fig. 2: Tübitak Uzay's sampling strategy for one $300 \mathrm{~m} \mathrm{x}$ $100 \mathrm{~m}$ areas

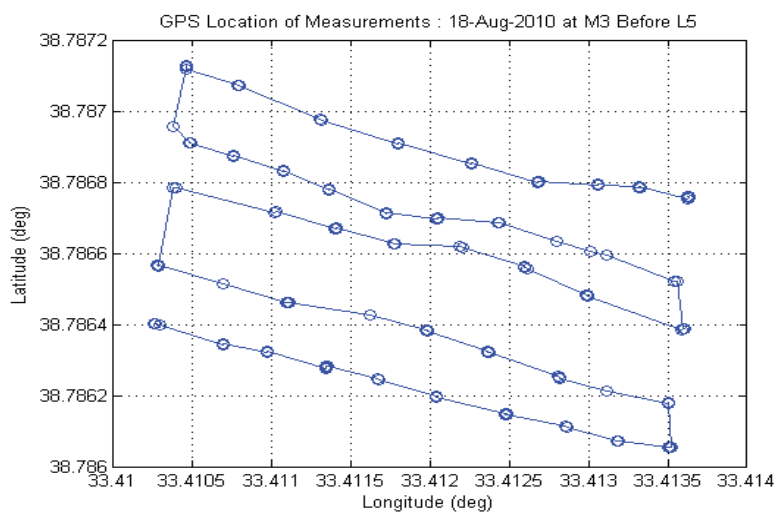

Fig. 3: CSIR's sampling strategy for one $300 \mathrm{~m} \mathrm{x} 100 \mathrm{~m}$ area

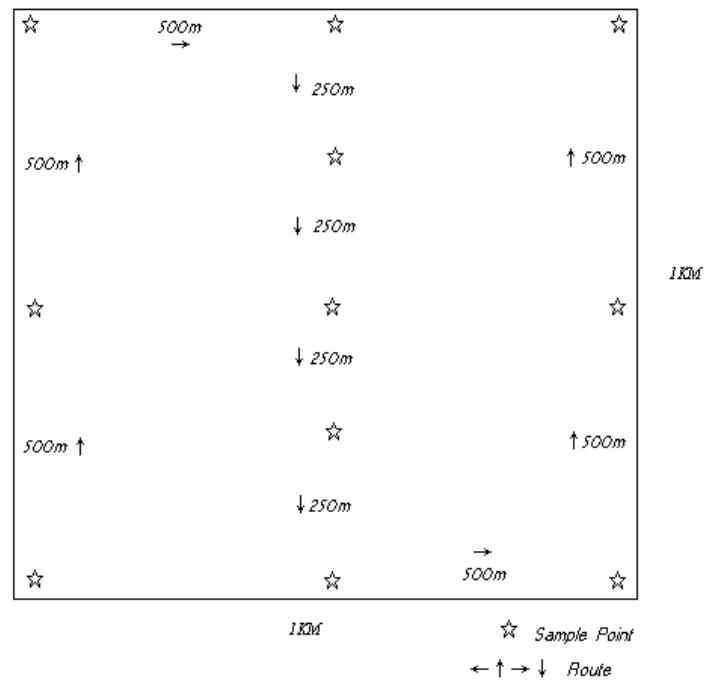

Fig. 4: CMA's sampling strategy for the $1 \mathrm{~km} \times 1 \mathrm{~km}$ area

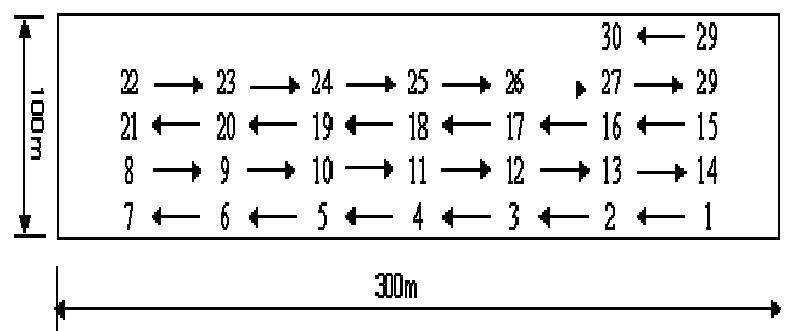

Fig. 5: INPE's sampling strategy for one $300 \mathrm{~m} \times 100 \mathrm{~m}$ area

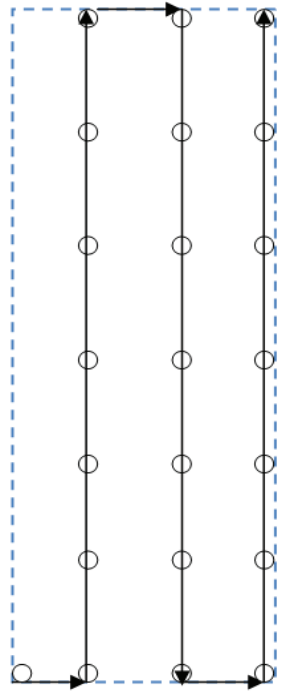

(a)

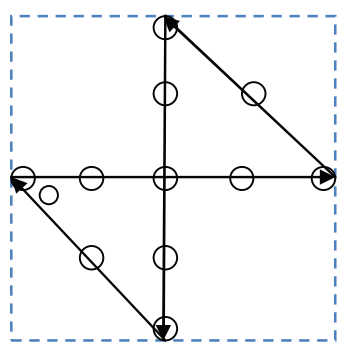

(b)

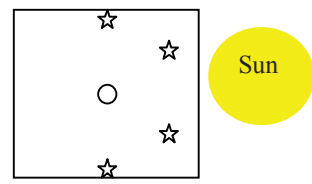

(c)
Fig. 6: VITO's sampling strategy for the $300 \mathrm{~m} \mathrm{x} 100 \mathrm{~m}$ areas (a), the $1 \mathrm{~km} \times 1 \mathrm{~km}$ area (b), and for each sampling point (c)

\subsection{In-motion sampling}

The "In-motion" technique to characterize an absolute calibration test site was developed by University of Arizona and South Dakota State University. Its philosophy is to keep the ASD spectrometer operator moving continuously. The methodology developed out of the need to characterize a site quickly to keep the impacts of BRDF and changing atmospheric effects insignificant. The site is broken up into equally spaced transects with the number dependent on size and time, preferentially oriented north/south which helps to minimize problems due to operator shadowing of the target. The aim is to record as many measurements as possible, but to complete the field characterization in less than one hour. The process initiates with a white panel optimization and measurement. Following this, one transect is walked, taking continuous radiance measurements of the surface, as the operator moves. The fiber optic is attached to a pole extended away from the operator at a height of approximately $1.5 \mathrm{~m}$. This allows for each "single" 
measurement to be averaged over a surface area of about $0.10 \times 3 \mathrm{~m}$, thus minimizing the effects of spatial nonuniformities. This positioning also minimizes the effects of stray light reflected off the operator as well as shadowing. The measurements continue as the operator returns along an adjacent transect. The white panel is moved to meet the operator on the adjacent transect. A second white panel is collected and the process is repeated until all transects are measured. Lastly after every 2-3 round trips, the ASD is reoptimized.

This methodology had also been used by CSIR, but with more frequent reference measurements (Fig. 8), and by Onera for the $1 \mathrm{~km} \times 1 \mathrm{~km}$ area. The ASD and its tripod were fixed to tricycle, the acquisitions being done by the biker every $15-20 \mathrm{~m}$ on the transect, and the trajectory of the bike recorded by GPS.

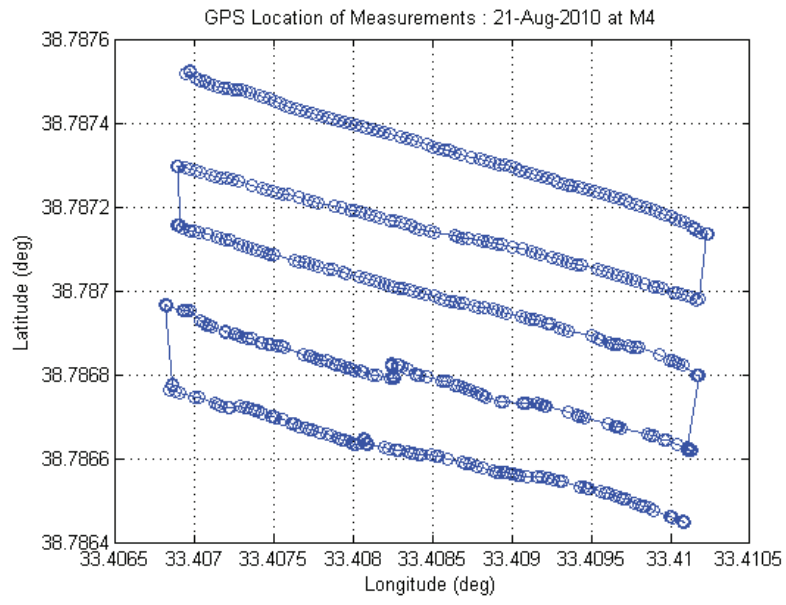

Fig. 8: CSIR's alternative in-motion sampling strategy

\section{COMPARISON OF THE METHODS AND DISCUSSION}

A $50 \mathrm{~m} \times 3 \mathrm{~m}$ strip was measured by all teams over a time frame of 15 minutes on 25 Aug 2010, allowing the comparison of different reflectance measurement methodologies for the same illumination conditions (sun zenith angle was $44.34^{\circ}$ ). Each participant measured this site in approximately 2 to 3 minutes, where the reference panel was sampled in the beginning and in the end of the stripe sampling. Each team sent his RF of this site and the associated standard uncertainty Type A and Type B to the pilot, NPL. In addition, the reported data included the RF and the associated standard deviation for each sampling point, when the team sampled the site using the space out sampling strategies, as described in the previous section. These sampling point values were further used by NPL to recalculate the RF of the site as a weighted mean of the individual sampling points values and the associated standard uncertainty to this weighted RF value following the GUM [4] recommendations.

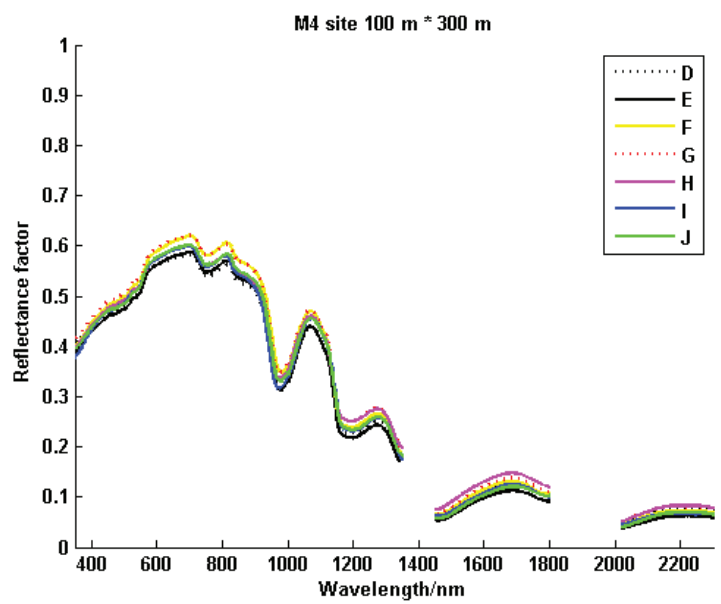

Fig. 9: RF measured by the participants on M4 area

The results of this comparison are still preliminary, since some data have to be recalculated. One of these preliminary results on a $300 \mathrm{~m} \times 100 \mathrm{~m}$ area (referenced as M4) is presented on Fig 9 where the RF reported by each participant is plotted. The corresponding measurements had been done by each team at different dates between August $17^{\text {th }}$ and $25^{\text {th }} 2010$, but for similar solar zenith angles (between $30^{\circ}$ and $40^{\circ}$ ). Each laboratory data is represented by a different letter. The curves present the same spectral shape, the differences being interpreted by the measurement uncertainties, the temporal variability of the reflectance, slight directional effects (taking or not into account by the different teams). These different contributors cannot be easily unmixed. However, considering the difference of methodology in the measurements, the dispersion between the RF measured by the different teams is relatively small (less than 0.04).

As previously mentioned, some RF's are going to be recalculated in the near future, and a deeper analysis of the results will be performed.

\section{REFERENCES}

[1] P.N. Slater et al., "Reflectance- and Radiance-Based Methods for the In-Flight Absolute Calibration of Multispectral Sensors", Remote Sensing Environment, 22, pp.11-37, 1987.

[2] H. Ozen et al, "The Tuz Golu Field Campaign - an overview", TH4.T05.1, Vancouver 2011 IGARSS, Canada, 24-29 July 2011.

[3] P. N. Slater, "Radiometric considerations in remote sensing," Proceedings of the IEEE, vol.73, no.6, pp. 997- 1011, June 1985.

[4] BIPM, "Evaluation of measurement data - Guide to the expression of uncertainty in measurement", JCGM 100:2008 GUM 1995 with minor corrections, corrected version 2010. 